\title{
The colossal circumvention of the lung lesion during lung stereotaxy
}

\author{
T. Rachi ${ }^{*}$, N. Nakamura², T. Akimotoº, R. Parshuram ${ }^{1}$, K. Motegi ${ }^{3}$, \\ T. Someya ${ }^{1}$ \\ ${ }^{1}$ Department of Radiological Technology, National Cancer Center Hospital East, Chiba, Japan \\ ${ }^{2}$ Department of Radiation Oncology, National Cancer Center Hospital East, Chiba, Japan \\ ${ }^{3}$ Division of Radiation Oncology and Particle Therapy, Research Center for Innovative Oncology, National Cancer \\ Center Hospital East, Chiba, Japan
}

\section{- Case report}

\section{*Corresponding authors:}

Toshiya Rachi, M.Sc.

E-mail: trachi@east.ncc.go.jp

Revised: October 2019

Accepted: October 2019

Int. J. Radiat. Res., October 2020; 18(4): $913-916$

DOI: 10.18869 /acadpub.ijrr.18.4.913

\begin{abstract}
This is a case report on stereotaxic (Stereotactic Body Radiotherapy-SBRT) for lung cancer located in the left lower lobe (Segment 6, S6). There have been no reports on marked displacement of the peripheral lung cancer during radiotherapy. A pulmonary nodule was discovered on computed tomography (CT) conducted for a persistent cough in an 87-year-old male. According to diagnostic imaging, this nodule was clearly delineated and had an irregular margin. The image diagnosis was T1NOMO, stage I primary lung cancer located in the left lower lobe and no pathological type was identified. The patient was treated with SBRT using a Linear accelerator (LINAC) at a total dose of 48 Gray (Gy) in 4 fractions. On performing cone beam CT (CBCT) at the third fraction, the tumor position had moved caudally by about 3 centimeters $(\mathrm{cm})$. For this reason, we canceled further treatment and the case was re-planned. Four days after discontinuing treatment, 4-dimensional CT (4DCT) images were obtained before initiation of the remaining fractions of SBRT. Therefore, the patient completed 4 fractions of SBRT and the tumor location was confirmed before beam delivery. The tumor location differed based on the patient's position (i.e., standing versus lying in a supine position), and we considered that it moved due to adhesion to the pleura when the patient was standing. If reproducibility of the tumor position cannot be guaranteed, the patient should undergo to $\mathrm{CT}$ re-simulation. Oncologists should re-evaluate the movement of the tumor on respiration and adjust the margins accordingly.
\end{abstract}

Keywords: SBRT, CBCT, 4-dimensional CT, Re-planning, adhesion, displacement.

\section{INTRODUCTION}

In general, Image Guided Radiation Therapy (IGRT) is performed before irradiation in Lung SBRT, and the tumor position is confirmed (1). The analysis of the change of the tumor position caused by respiration using the image acquired by IGRT and countermeasures are often studied. Many studies have been conducted on variations and corrections in the tumor location due to breathing or related to patient movement (2).
However, there are only limited cases where the tumor position has changed due to other factors. Therefore, there are almost no countermeasures or examinations.

In the present case, the tumor position shifted unexpectedly during the treatment period, regardless of breathing or patient movement. The purpose of this case report is to identify the cause of the change in the location of the tumor and suggest the best subsequent treatment. 


\section{Case presentation}

An 87-year-old male was noted to have an abnormal lung nodule on the left lower lobe. Diagnostic contrast-enhanced computed tomography (CECT) of the thorax detected a nodular lesion measuring $23 \times 29 \times 25 \mathrm{~mm}$ (Anterior-Posterior $\times$ Medio-Lateral $\times$ SuperiorInferior) in the peripheral region of the lower left lobe S6. 18-Fluoro-2-deoxyglucose positron emission tomography (FDG-PET) showed an FDG avid lesion (SUV Max-14.22). PET images are presented in figure 1 . Ultimately, the patient was diagnosed with left lower lobe lung cancer, clinical stage T1cN0M0 (Unio Internationalis Contra Cancrum 8th) without pathological confirmation, and lung stereotaxy was planned after a discussion with the multidisciplinary care team (MDT) (3).

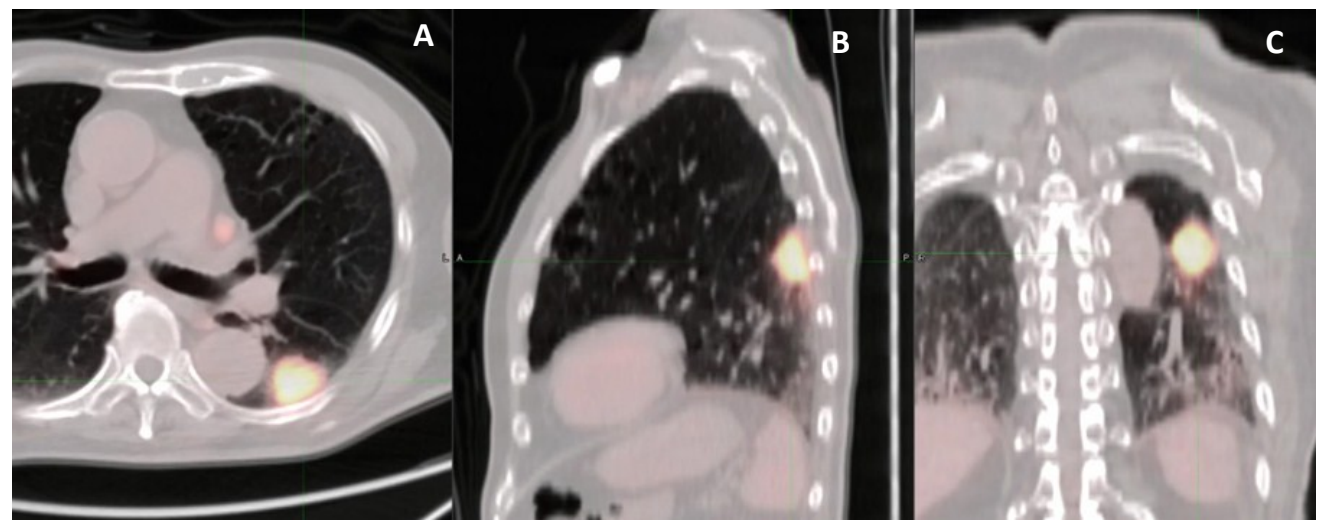

Figure 1. FDG-PET images before SBRT (SUV Max-14.22). A nodular lesion is examined in the left lower lobe S6. A: PET axial image, B: PET sagittal image, C: PET coronal image.

The patient was treated with SBRT on LINAC (True Beam; LINAC from Varian Medical Systems) at a dose of 48 Gy in 4 fractions [biological effective dose (BED) $10=105.6 \mathrm{~Gy}$ ] (4). 4DCT with an added respiratory phase was performed to determine the internal target volume (ITV) (5). The dose distribution was calculated using a three-dimensional radiation treatment planning system (Eclipse from Varian Medical Systems). The clinical target volume (CTV) was defined as the same volume as the gross tumor volume (GTV). The planning target volume (PTV) margin was defined by considering the internal margin in CTV. Additional margins were as follows: add $6 \mathrm{~mm}$ to the right side, $5 \mathrm{~mm}$ to the left side, $5 \mathrm{~mm}$ to the ventral side, $5 \mathrm{~mm}$ to the dorsal side, $5 \mathrm{~mm}$ to the head side, and $10 \mathrm{~mm}$ to the foot side $(5,6)$. Normal organs including the spinal cord, esophagus, lung, trachea, and main bronchus contoured and were considered to be organs at risk (OAR). PTV was constrained so that the volume surrounded by $95 \%$ or more of the prescribed dose would be $100 \%$ (7). Figure 2 shows the dose distribution. Set-up verification of the patient before SBRT was performed using CBCT, after which X-ray images in two directions were acquired on exhalation using On-Board Imaging (OBI) and an Electronic Portal Imaging Device (EPID) (8). Because the tumor showed respiratory movement of $5 \mathrm{~mm}$ or more, SBRT was performed during exhalation using a respiratory gating system. CBCT was performed again to confirm the intra-fractional variation during irradiation.

For the first and second fractions, displacement of the intra-fractional variation was within $5 \mathrm{~mm}$ of the maximum (9), and treatment could be completed. However, CBCT prior to the third fraction revealed tumor displacement to the caudal side by over $3 \mathrm{~cm}$ as compared with the position on treatment planning CT (figure 3). As a result, SBRT for that day was canceled. CT re-simulation was performed and treatment was re-planned. At this time, the tumor size did not change, and the same margins as noted above were added. Respiratory movement of the tumor was within

Int. J. Radiat. Res., Vol. 18 No. 4, October 2020 
$5 \mathrm{~mm}$, and SBRT after re-planning was performed under natural breathing. The patient underwent 4DCT before SBRT to confirm the tumor position and respiratory movement. CBCT performed just prior to the third fraction of SBRT showed no change in the tumor position from the time of re-planning. The intrafractional variation was within $5 \mathrm{~mm}$ of the maximum, and SBRT could be performed with good reproducibility. The fourth fraction was performed in a similar tumor position, and SBRT was completed.

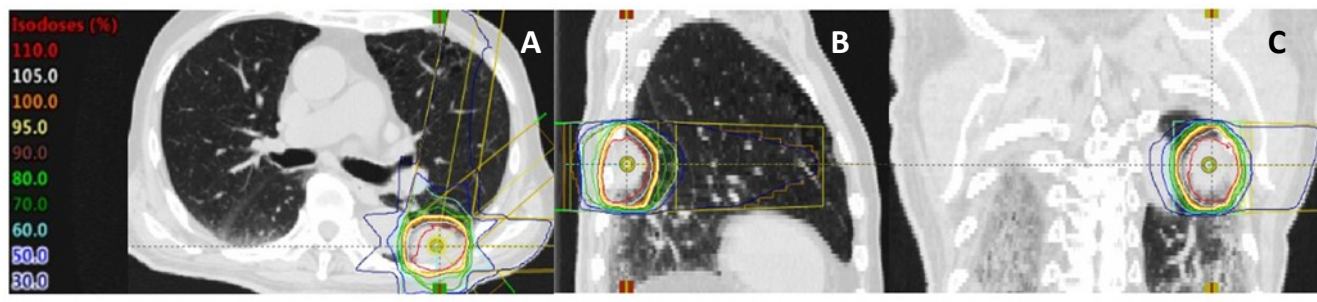

Figure 2. Dose distribution at the left lower lung S6.

A: Dose distribution from axial image, B: Dose distribution from sagittal image, C: Dose distribution from coronal image.

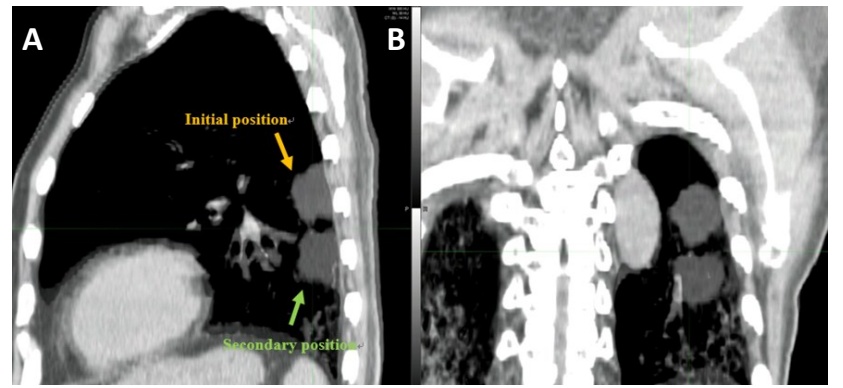

Figure 3. Fusion image showing initial planning $\mathrm{CT}$ and second re-planning $\mathrm{CT}$.

A: Change in tumor position viewed from axial image, B: Change in tumor position viewed from coronal image.

\section{DISCUSSION}

We reported an unusual case of a lung tumor located in the left lower lobe that was displaced caudally by over $3 \mathrm{~cm}$ during the lung SBRT period.

Many have reported on accuracy management and measures against respiratory movement in SBRT for the tumor misalignment $(1,2,5)$. Nakaguchi et al. reported the high accuracy of MU to prescription points and invariance to tumor location when performing respiratory synchronization in SBRT (10). Caillet et al. reported the IGRT technique to ensure the effect of SBRT and reduce toxicity (8). Also marked tumor evasion in CRT for as a case report similar to ours, Grant SR et al. reported esophageal cancer (11). However, our report is the first to describe a tumor location that was significantly displaced during the SBRT period, regardless of factors such as breathing or body movement reported in previous studies.
First of all, we estimated that approximately $3-\mathrm{cm}$ displacement from the original tumor position during treatment was within the normal range of changes due to respiration. The Real-Time Position Management (RPM, Varian Medical Systems) system was used in treatment planning CT and the total respiratory phase and 4DCT were recorded. As a result, the tumor position in the first half of SBRT had moved markedly with movement of the diaphragm. Because the position of the tumor in the latter half did not move at all in accordance with respiration, it is not considered to be a shift due to respiration. Therefore, it is unlikely that the tumor would have been displaced caudally by changes in respiration.

Another explanation for the displacement is tumor adhesion to the pleura after the second fraction of SBRT. The tumor position during standing differed from that in the supine position, with the former showing a caudal tumor position. Figure 4 shows computed 
radiography (CR) images before SBRT treatment and two coronal plane images reconstructed from the initial and re-planned CT images. Comparison of these images revealed the tumor position in the CR image taken in the standing position to be the same as the tumor position in the re-planned CT image. This suggests that the tumor may have adhered to the pleura when the patient was standing after the second fraction. The amount of movement by respiration would have decreased in the second half of SBRT due to adhesion of the tumor to the pleura.

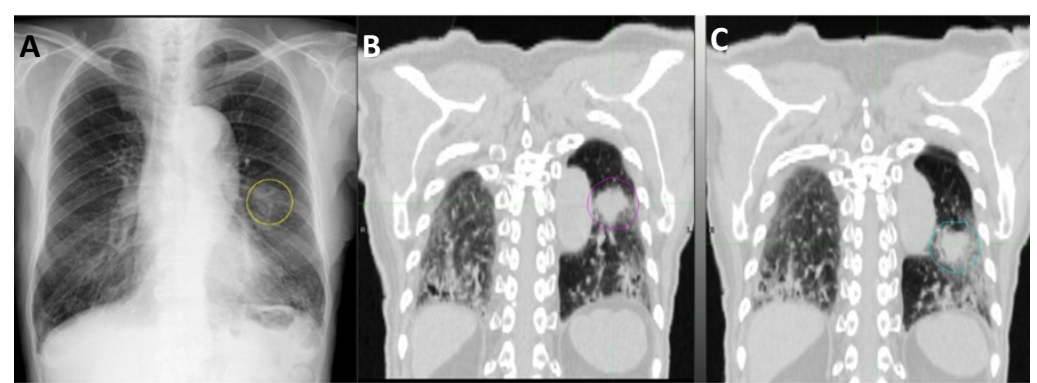

Figure 4. A: CR image before treatment is started, B: Coronal images of initial planning CT, C: Coronal images of re-planning CT.

\section{CONCLUSION}

This paper describes the colossal displacement of a lung tumor during lung stereotaxy. We reconfirmed the importance of performing re-planning 4DCT and CBCT for such cases. Also, it is necessary to keep in mind that the position of the tumor may be vary depending on the patient's positioning.

\section{ACKNOWLEDGEMENTS}

We thank all colleagues who helped us write this case report. We are also grateful to the patient.

\section{Ethical approval}

There is no need for ethical review of case reports as per our institutional regulations. However, consent has been obtained from the patient himself in this case.

\section{Conflicts of interest: Declared none.}

\section{REFERENCES}

1. Bissonnette JP, Purdie TG, Higgins JA, et al. (2009) Conebeam computed tomographic image guidance for lung cancer radiation therapy. Int J Radiat Oncol Biol Phys, $\mathbf{7 3}$ (3): 927-34.
2. Lu L, Diaconu C, Djemil T, et al. (2018) Intra- and interfractional liver and lung tumor motions treated with SBRT under active breathing control. J Appl Clin Med Phys, 19 (1): 39-45.

3. Onishi H, Shirato H, Nagata Y, Hiraoka M, et al. (2011) Stereotactic Body Radiotherapy (SBRT) for Operable Stage I Non-Small-Cell Lung Cancer: Can SBRT Be Comparable to Surgery? Int J Radiat Oncol Biol Phys, 81(5): 1352-1358.

4. Jones B, Cominos M, Dale RG (2003) Application of biological effective dose (BED) to estimate the duration of symptomatic relief and repopulation dose equivalent in palliative radiotherapy and chemotherapy. Int J Radiat Oncol Biol Phys, 55(3): 736-42.

5. Iris E van Dam, John R van Sörnsen de Koste, Gerard G. Hanna, Rebecca Muirhead, et al. (2010) Improving target delineation on 4-dimensional CT scans in stage I NSCLC using a deformable registration tool. Int J Radiat Oncol Biol Phys, 96(1): 67-72.

6. Persson GF, Nygaard DE, Hollensen C, Munck af Rosenschöld P, et al. (2012) Interobserver delineation variation in lung tumour stereotactic body radiotherapy. $\mathrm{Br}$ J Radiol, 85: 1017.

7. Ghandour S, Cosinschi A, Mazouni Z, Pachoud M, Matzinger $\mathrm{O}$, et al. (2016) Optimization of stereotactic body radiotherapy treatment planning using a multicriteria optimization algorithm. Z Med Phys, 26(4): 362-370.

8. Caillet V, Booth JT, Keall P (2017) IGRT and motion management during lung SBRT delivery. Phys Med, 44: 113122.

9. Josipovic M, Persson GF, Logadottir A, et al. (2012) Translational and rotational intra- and inter-fractional errors in patient and target position during a short course of frameless stereotactic body radiotherapy. Acta Oncol, 51 (5): 610-7.

10. Nakaguchi Y, Araki F, Kouno T, et al. (2012)Quality assurance of respiratory-gated stereotactic body radiation therapy in lung using real-time position management system. Nihon Hoshasen Gijutsu Gakkai Zasshi, 68(11): 1519-24.

11. Grant SR, Page CM, Diaz I, et al. (2018) The great esophageal escape: A case of extreme esophageal interfraction motion during neoadjuvant chemoradiation therapy. Pract Radiat Oncol, 8(5): 251-254.

Int. J. Radiat. Res., Vol. 18 No. 4, October 2020 